\title{
Arch replacement for acute type A aortic dissection using a single fenestrated frozen elephant trunk technique
}

\author{
Homare Okamura, Mamoru Arakawa, Atsushi Miyagawa, Yuichiro Kitada, Hideo Adachi \\ Department of Cardiovascular Surgery, Nerima Hikarigaoka Hospital, Tokyo, Japan \\ Correspondence to: Homare Okamura, MD, PhD. Department of Cardiovascular Surgery, Nerima Hikarigaoka Hospital, 2-11-1 Hikarigaoka, Nerima- \\ Ku, Tokyo 179-0072, Japan. Email: homareo@jadecom.jp.
}

Submitted Nov 25, 2019. Accepted for publication Feb 04, 2020. doi: $10.21037 /$ acs.2020.02.03

View this article at: http://dx.doi.org/10.21037/acs.2020.02.03

\section{Clinical vignette}

A 42-year-old man who presented with back pain and paralysis of the left lower extremity was diagnosed with acute type A aortic dissection and transferred to our hospital for surgery. His past medical history included hypertension; however, he denied a family history of aortic disease. Contrast-enhanced computed tomography (CT) revealed type A aortic dissection with an entry tear in the transverse aortic arch and compression of the true lumen of the abdominal aorta. The patient's vital signs were stable, however muscular weakness of the left lower extremity was noted. Transthoracic echocardiography revealed preserved left ventricular function without aortic regurgitation.

\section{Surgical technique}

\section{Preparation and exposure}

The patient was placed in a supine position following induction of general anesthesia. Bilateral radial and femoral arterial lines were placed, and transesophageal echocardiography (TEE) was performed. Median sternotomy was performed, and cardiopulmonary bypass $(\mathrm{CPB})$ was established via cannulation of the ascending aorta, the right atrium, and a left ventricular vent. After initiation of $\mathrm{CPB}$, blood pressure reduced to an immeasurable level in the right lower extremity, necessitating arterial cannulation of the right femoral artery.

\section{Operation}

The supra-aortic vessels were exposed and mobilized during systemic cooling. Hypothermic circulatory arrest was performed at a rectal temperature of $28{ }^{\circ} \mathrm{C}$. Cardioplegic arrest was achieved with the intermittent administration of retrograde and antegrade cold blood cardioplegia. Selective antegrade cerebral perfusion was initiated during the period of circulatory arrest with insertion of catheters into the innominate and left common carotid arteries, and the entry tear in the transverse arch was excised. The left subclavian artery (LSCA) was clamped. The transverse arch was transected proximal to the LSCA, and a commercially available frozen elephant trunk (FET) (J Graft FROZENIX, Japan Lifeline, Tokyo, Japan) was deployed into the descending aorta in an antegrade fashion. The FET stent graft size was selected based on $90 \%$ of the diameter of the descending aorta on preoperative CT. The FET of an optimal length was selected to ensure that the distal end of the FET was placed in the straight segment of the descending aorta. The diameter and length of the FET were 27 and $120 \mathrm{~mm}$, respectively. Subsequently, a hole (approximately $10 \mathrm{~mm}$ ) was manually created on the LSCA aspect of the FET. Before performing the distal anastomosis, the fenestrated FET graft was fixed to the aorta to prevent endoleak through the fenestration. A piece of a 4-branched J graft (Japan Lifeline, Tokyo, Japan) was tailored into a $U$ shape and placed around the LSCA. Transmural fixation of the U-shaped graft piece to the FET within the aorta was performed using continuous 4-0 polypropylene suture. After reinforcement of the distal end of the aorta with a felt strip, the 4-branched graft was anastomosed to the distal end using running 4-0 polypropylene sutures. Systemic perfusion was restored through the side branch of the graft. Total circulatory arrest 
time was 33 minutes. Subsequently, proximal anastomosis was performed using continuous 4-0 polypropylene sutures with double-felt-strip reinforcement. With the heart beating, reconstruction of the left common carotid and innominate arteries was performed using continuous 5-0 polypropylene sutures. The CPB and aortic cross-clamp times were 199 and 77 minutes, respectively.

\section{Completion}

Blood pressure readings did not differ between the patient's arms, thereby confirming adequate blood flow through the LSCA. TEE revealed expansion of the true lumen of the distal aorta. Thereafter, the chest was closed in the usual fashion.

The patient's postoperative course was uneventful. Three-month postoperative CT revealed well-preserved blood flow into the LSCA through the fenestration without endoleak. We observed a well-expanded true lumen in the downstream aorta with a thrombosed false lumen.

\section{Comments}

FET implantation promotes thrombosis of the false lumen in the distal aorta and prevents reoperation in patients undergoing aortic repair for acute type A aortic dissection (1). However, total arch replacement using the FET technique is technically challenging, particularly for surgeons with less experience in aortic surgery.

The fenestrated FET technique allows the surgeon to perform the distal anastomosis more proximally within the boundaries of the transverse aortic arch without LSCA reconstruction. In this case, the fenestration was accurately and easily created at the LSCA orifice under direct vision. The use of ascending aortic cannulation ensured that all procedures could be performed via median sternotomy without an additional axillary or groin incision.

Since the introduction of the fenestrated FET technique (2), we have used the following modifications to prevent kinking of the FET and endoleak through the fenestration. The Frozenix FET prosthesis comprises a stent and a graft portion (3). Previously, we left behind $2 \mathrm{~cm}$ of the graft portion of the FET for distal anastomosis. However, a longer graft portion increases the risk of FET kinking in patients with a steeply angulated transverse arch. Therefore, our modification includes creation of the distal anastomosis on the stent portion of the FET without the graft portion. Moreover, we avoid the fenestrated FET technique in patients with a significantly steep angulation of the aortic arch. Our second modification involves the method of fixation of the fenestrated FET into the aorta. Endoleak formation invariably complicates the fenestration technique, and the risk of delayed endoleak-induced aneurysm formation in the proximal descending aorta cannot be excluded. Therefore, our modification includes fixation of the FET using continuous 4-0 polypropylene sutures in close proximity to the fenestration to prevent endoleak through the fenestration. A fenestrated FET technique reported by Roselli et al. (4) includes stenting of the LSCA via the fenestration to prevent endoleak. Our fixation technique with direct suture is technically easy and ensures secure graft fixation, and additional stenting of the LSCA is not required.

The fenestrated FET technique is feasible in most patients undergoing aortic repair for acute type $\mathrm{A}$ aortic dissection; however, it should be avoided in patients with a steeply angulated aortic arch or in those with an entry tear located at the origin of the LSCA. Our technique successfully prevents blood flow into the entry tear and is safe in patients with an entry tear located in the lesser curvature of the transverse aortic arch.

In conclusion, the fenestrated FET technique is a simple and effective procedure to treat acute type A aortic dissection. In addition to expediting the aortic repair procedure, it facilitates easier arch repair in cases of aortic dissection.

\section{Acknowledgments}

None.

\section{Footnote}

Conflicts of Interest: The authors have no conflicts of interest to declare.

Open Access Statement: This is an Open Access article distributed in accordance with the Creative Commons Attribution-NonCommercial-NoDerivs 4.0 International License (CC BY-NC-ND 4.0), which permits the noncommercial replication and distribution of the article with the strict proviso that no changes or edits are made and the original work is properly cited (including links to both the formal publication through the relevant DOI and the license). See: https://creativecommons.org/licenses/by-ncnd/4.0/. 


\section{References}

1. Dohle DS, Tsagakis K, Janosi RA, et al. Aortic remodelling in aortic dissection after frozen elephant trunk. Eur J Cardiothorac Surg 2016;49:111-7.

2. Okamura H, Arakawa M, Takeuchi T, et al. The fenestrated frozen elephant trunk technique for acute type A aortic dissection. J Thorac Cardiovasc Surg

Cite this article as: Okamura H, Arakawa M, Miyagawa A, Kitada Y, Adachi H. Arch replacement for acute type A aortic dissection using a single fenestrated frozen elephant trunk technique. Ann Cardiothorac Surg 2020;9(3):248-250. doi: 10.21037/acs.2020.02.03 2018;156:e75-7.

3. Uchida N, Katayama A, Higashiue S, et al. A new device as an open stent graft for extended aortic repair: a multicenter early experience in Japan. Eur J Cardiothorac Surg 2016;49:1270-8.

4. Roselli EE, Tong MZ, Bakaeen FG. Frozen elephant trunk for DeBakey type 1 dissection: the Cleveland Clinic technique. Ann Cardiothorac Surg 2016;5:251-5. 